reconfirmed by MR angiography in 6 patients; CA Doppler scanning was used alone in 4 patients. Thirty patients were treated with medical therapy or observation. Open surgical or endovascular treatment of CAD was rarely employed in the pediatric population. Of 17 patients treated conservatively with anticoagulation/antiplatelet therapy, 4 recovered completely, and 11 were partially recovered. Of 12 patients treated expectantly without anticoagulation, 2 recovered completely, 8 recovered partially, and 2 died. Of 4 patients treated surgically, 3 showed partial improvement, and 1 died 5 days postoperatively. The authors favor MR angiography as a screening test, anti-platelet therapy to anticoagulation therapy as first-line medical treatment, and endovascular stenting over open surgery in cases of failed medical treatment. (Chamoun RB, Mawad ME, Whitehead WE, Luerssen TG, Jea A. Extracranial traumatic carotid artery dissections in children: a review of current diagnosis and treatment options. J Neurosurg Pediatrics Aug 2008;2:101-108). (Respond: Andrew Jea MD, Dept Neurosurgery, Division of Pediatric Neurosurgery, Texas Children's Hospital, Baylor College of Medicine, Houston, TX. E-mail: ajea (a bem.tmc.edu).

COMMENT. An algorithm for the evaluation and treatment of traumatic extracranial carotid artery dissection is proposed, based on a review of the literature. Arterial dissection is identified as the fourth most common cause of arterial ischemic stroke in children, after sickle cell disease, cardiac embolism, and moyamoya disease.

\title{
THORACOLUMBAR SPINE SUBDURAL HEMATOMA IN INFANT
}

A thoracolumbar subdural hematoma (SDH) resulting from nonaccidental trauma in a 4-month-old infant is reported from Women and Children's Hospital, University of Buffalo, NY. The patient was noted to be apneic and had been shaken by a parent in an attempt to stimulate respiration. In the ED he was unresponsive and was diagnosed with myelopathy. In addition to bilateral clonus and extensor plantar responses, he had a bulging fontanelle, bruising on left shoulder, and retinal hemorrhages. Head CT showed SDH and subarachnoid hemorrhage. MRI of spine showed a SDH dorsal to the spinal cord with mass effect on the conus medullaris and descending nerve roots. Emergency T-12 to L-3 laminectomies were performed, and the hematoma was evacuated. Later, he required a ventriculoperitoneal shunt, and currently, he has severe developmental delay. (Gruber TJ, Rozzelle CJ. Thoracolumbar spine subdural hematoma as a result of nonaccidental trauma in a 4-month-old infant. $\mathbf{J}$ Neurosurg Pediatrics Aug 2008;2:139-142). (Respond: Curtis J Rozzelle MD, University of Buffalo Neurosurgery, Women and Children's Hospital of Buffalo, 219 Bryant Street, Buffalo, NY, 14222. E-mail: crozzelle (a.kaleidahealth.org).

COMMENT. Spinal cord injury is an uncommon complication of shaken baby syndrome, and may be overlooked when associated with head trauma. The authors explain the injury as a result of hyperflexion and hyperextension of the spine around the rigid rib cage. It is important to perform imaging of the spine, in addition to head and neck in infants with shaken baby syndrome. 